\title{
Emotional augmented reality-based mobile learning design elements: a kansei engineering approach
}

\author{
Fauziah Redzuan, An-Nur Atiqah Khairuddin, Nor Aziah Daud \\ Faculty of Computer and Mathematical Sciences, Universiti Teknologi MARA (UiTM), Malaysia
}

\begin{tabular}{l} 
Article Info \\
\hline Article history: \\
Received Oct 21,2018 \\
Revised Dec 1, 2018 \\
Accepted Dec 14, 2018 \\
\hline
\end{tabular}

\section{Keywords:}

Augmented Reality

Design elements

Emotion

Kansei Engineering

Mobile Learning

\begin{abstract}
In recent times, various studies have shown that Augmented Reality (AR) will be the next wave of online learning. This is because of the advent of powerful smartphones that has changed user experiences, thereby able to increase the capability of AR. There has been much concentration in previous studies on cognition towards the use of AR in education, in which little consideration has been given to emotions which is also an important aspect in learning. Based on this, the present research aims to identify salient connections between emotions and design elements of AR-based mobile learning material through the application of the Kansei Engineering (KE) approach. In order to achieve this study objective, the use of a human heart in relation to the mobile AR application of the KE approach was adopted in this research as a case study, in which seven specimens of the mobile AR application were evaluated including 55 emotions of Kansei Words (KW). Additionally, the kansei evaluation experiment of this study was carried out by 28 students from one of the public universities, after which the data were analysed using Factor and Principal Component Analysis. The results of this study show the important pillars of emotions or Kansei semantic space of emotions for AR-based mobile learning materials. Based on Factor Analysis, it revealed four main pillars; professional-motivated, confused, wanderingthrilled, challenging and one additional pillar; trustable. Besides that, this research also described design elements of AR-based mobile learning material that might evoke specific emotions based on the identified pillars. Finally, the findings of this research are hoped to be applicable as a guide in design during preparation of AR-based mobile learning materials with affective elements in the future.
\end{abstract}

Copyright @ 2019 Institute of Advanced Engineering and Science. All rights reserved.

Corresponding Author:

Fauziah Redzuan,

Faculty of Computer and Mathematical Sciences,

Universiti Teknologi MARA (UiTM),

40450 Shah Alam, Selangor, Malaysia.

Email: fauziahr@tmsk.uitm.edu.my

\section{INTRODUCTION}

This paper presents the research on emotional Augmented Reality (AR)-based mobile learning design elements based on Kansei Engineering (KE) approach. Currently, the Internet penetration in Malaysia stands at $67 \%$, thereby making it reliable to harness the power of e-learning for online global education [1]. Even with the persistent increase of online course enrolment, there still exist serious issues contributing to its low retention rates [2]. Among such factors are; educational level, failure in understanding the content, students' satisfaction, and students' motivation [2-4]. The preliminary study shows that most students have difficulty in learning complex courses with abstract concepts, and are less satisfied with the current online learning materials. 
Furthermore, it has been proven that Augmented Reality (AR) is the future of e-learning [5,6]. A comprehensive survey by [7] presented that AR can be used for online learning, thereby able to provide effective learning. Currently, there has been a rapid trend development for AR applications and the adoption of mobile computing devices, such as smartphones and tablets [8,9]. This is because the aids of multimedia elements, and interaction in the design of AR vis a vis the abstract concept can facilitate learning [10,11]. However, education oriented of AR applications has not been so deeply explored [12]. Reason being that, previous researches related to AR only focused on cognition while neglecting the emotional or affective value in learning [13].

Consequently, the delivery of the right content to learners through e-learning coupled with good design will ultimately result into an effective learning [14,15]. Moreover, researchers believed that the emotions of student during course engagement play a vital role in any learning environment, including in elearning [16,17]. Recently, some researchers have explored progressively on student's emotion in e-learning, especially in higher education, but unfortunately studies that capture student's emotion in e-learning are still lacking, even though the understanding of learner's emotion is important in order to design the learning material [18].

Generally, AR can be described as a multidisciplinary field that encompasses computer graphics, vision and multimedia, which deals with the real-time combination of digital (computer-generated data) and physical information (real world) through different technological devices [19]. Mobile AR (MAR), on the other hand extends the scope and prospective functionality of AR, thereby presenting a dynamic procedure for people to interact with computers and digital information [20].

Findings in most studies have indicated the effectiveness of AR in learning, such as enhancing learning performance through abstract concept as mentioned in the study of [21], and promoting learning motivation as well as increasing learning engagement [22]. Despite the benefits highlighted in previous studies, education oriented AR applications have not yet been deeply explored [23]. As mentioned earlier, AR can be integrated with e-learning, thus making it the next wave in online learning [24,25].

One of the characteristics of AR is the integration and interaction between the real and virtual world, which allows huge versatility and creativity in learning [26]. Not only the learners able to see and listen the virtual information, they can also manipulate and also able to repeat specific part of the augmentation which can benefit in their learning experience [27]. According to [28] there are two types of contents deployed in an AR application; (i) static (texts, 3D model) and (ii) dynamic (animation). Generally, the interface interaction between the students and the device used in online learning is made by the mean buttons or gestures because students can move the 3D object with buttons, scale the size of the video, image or object, or play a video $[29,30]$. A study by [31] classified five usability principles for smartphone AR applications, which are; userinformation, cognitive, support, interaction, and usage respectively.

As stated before, there is still very few research that look into the role of emotions with AR technology to reinforce learning. A few examples can be found in [30] and [32], in which both studies revealed that most of the students enjoyed learning difficult course (abstract or technical course) through the use of AR. In reference to [33], suggested that designers of a program application may manipulate the properties of the artefact to trigger a desired emotional state, and should not neglect the importance of reinforcing positive emotions.

Over the year, there have been various studies carried out on the effectiveness of AR in learning, but there is still lack of empirical work that explores the role of emotions in supporting learning through the use of AR technology. A few examples of AR researches that have emphasized on emotions can be found in historical education [34], tourism industry [33] and computer science subject [32]. Recently, a study by [34] examined the role of academic achievement emotions by comparing virtual and location-based AR using AR historical tour mobile applications.

Kansei Engineering (KE) is defined as a technique of an ergonomic and consumer-oriented technology to translate user's Kansei (emotions, feelings, and demands) into a design elements [35,36]. In other words, $\mathrm{KE}$ is a technology which unites Kansei into engineering realms by providing a systematic procedure of understanding the insight of user perceptions about the artefacts in order to produce concrete design characteristics that match consumer's need and desire via several physiological and psychological measurement methods [37].

$\mathrm{KE}$ method has been applied in various fields, where it mainly aims at assisting designers to understand consumers' emotions and impressions to contribute solutions and help provide concrete design parameters [38]. Among the implementations of $\mathrm{KE}$ which focus on the design of physical product, particularly IT artefact are online clothing website [39], virtual reality [40], online learning [41], and videobased learning [42]. Therefore, in this study, KE method is employed, as it is the most suitable approach in order to meet the objective of this research. 
Therefore, the focus of this research is to engineer emotional experience in order to achieve an effective formula during preparation of AR mobile learning materials. The intention of this research is to provide answers to two objectives; (i) To identify the emotions of students during their experience with ARbased mobile learning materials and (ii) To identify the specific emotions associated with the AR-based mobile learning design elements. However this research only discusses the second objective as for the first objective has already been discussed in [43].

\section{RESEARCH METHOD}

In this research, KE methodology has been employed with the quantitative approach using psychological measurement. Overall, this research follows KE Type II procedure which is adopted from [44] and divided into two phases; 1 and 2 as in Figure 1. Also, literature reviews were done in this research in order to identify issues and problem that occurs in e-learning environment. In order to support issues and obtain information as well as opinions regarding e-learning, a preliminary investigation has been carried out with 32 students from a local public university using online survey. Afterwards, problem statement, research questions and research objectives were defined based on findings from both literature reviews and preliminary study. This was followed by the phase 1 of this study, which is to identify important emotions in e-learning environment. This resulted in the selection of 55 emotions adopted from a research carried out by [41]. Apart from that, these emotions were also used for phase 2 of the present research, in which the aim of the phase 2 is to identify the design elements for specific emotions which are the main focus of this research.

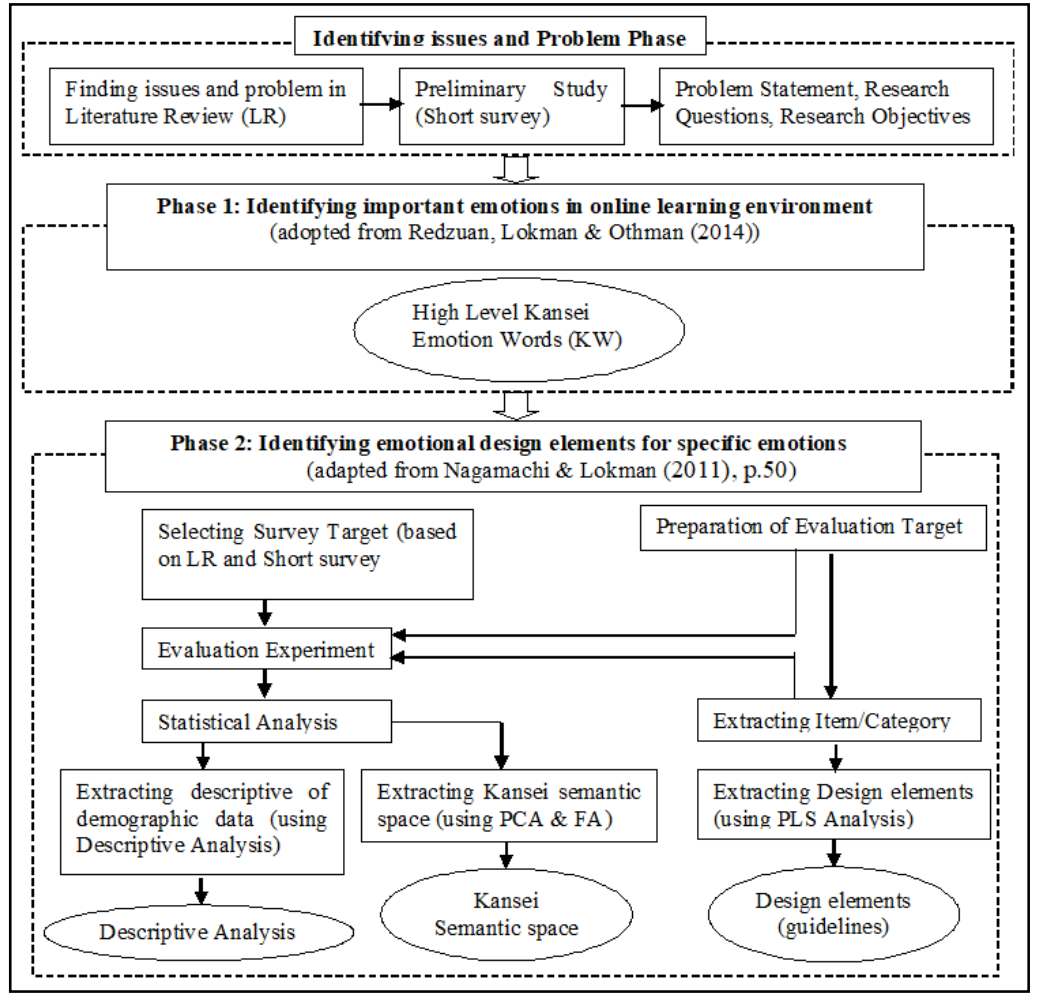

Figure 1. An overview of summarize research methodology steps using KE Type II method adapted from [44]

Therefore, phase 2 began with the selection of a survey target. The first step is purposely to determine the focus domain that was derived from the initial work in phase 1 . The following step was the preparation of the evaluation target which consists of three important aspects. Firstly, the specimen or AR of mobile learning material was examined. Based on this, seven specimens were prepared to focus on the human anatomy course, particularly the human heart topic. The decision was based on an earlier preliminary study and the observation activity of ex-isting applications in the current market. Results from the preliminary study revealed that the majority of the students have Android smartphone. The chosen topic (the heart) for this study was found to be among the highest topic that needs support for mobile learning and has more

Emotional augmented reality-based mobile learning design elements: a kansei... (Fauziah Redzuan) 
options of AR mobile learning materials. Therefore, four specimens of mobile-based application were obtained from Google Play Store, while the remaining three were generated through the development of The Human Heart Mobile AR application (HeMAR) which was developed in an android platform that used camera-based tracking method in visualizing the AR scene via marker. The functional requirement was identified from the observational activity, while user requirement was obtained from the preliminary study. Also, Unity and Vuforia platform were used to develop HeMARs, while user experience (UX) which encompasses human factor and usability as mentioned in [8] was taken as a basis. Based on these, all the chosen seven specimens have differences and similarities in design which were found to fit the selection criteria according to the rules of KE method.

Secondly, the subjects to participate in the evaluation experiment were selected through the use of purposive sampling in one of the public universities in Malaysia. The subjects or participants were undergraduate students who currently enrolled or had taken the human anatomy course. Moreover, the criteria for the participants are that they must be familiar with e-learning and able to express emotions accordingly. As stated by Nagamachi in [44], a number of 20 or 30 people are sufficient to be employed as subjects in KE research. Based on this, 35 students were invited for the evaluation in this study, but only 32 of them joined. Thirdly, Semantic Differential (SD) scale of the experiment was configured and 55 emotions as mentioned in phase 1 were used in 5-level SD scale for evaluation. Fourthly, an extracting item/category to investigate the design of the specimen (e.g. colour, size and layout) was carried out. In KE, the specific characteristics in product design are the item and the small groupings in each item are known as category. The evaluation experiment was conducted in a library room to provide a controlled environment. Analysis of the data collected from the evaluation experiment were conducted through three categories of analysis, which are; (i) descriptive analysis (for demographic profile), (ii) combination of Factor Analysis (FA) and Principal Component Analysis (PCA) (for Kansei semantic space), and (iii) Partial Least Square (PLS) (for design elements or guidelines).

\section{RESULTS AND ANALYSIS}

There are two findings expected in this research, which are (i) Kansei semantic space of AR-based mobile learning specifically for Human Anatomy learning materials for higher education, (ii) the design elements in association with a specific emotion. These findings were obtained from Factor Analysis (FA), Principal Component Analysis (PCA) and Partial Least Square (PLS) method in this study using the average evaluation data. The FA and PCA with varimax rotation were performed to identify the semantic space of AR-based mobile learning material. The FA findings show that, variance analysis result can be obtained to determine the most significant factors of emotion. In short based on the first objective the finding reveals the identified semantic space for AR-based mobile learning materials is structured based on five factors or pillars. The four main pillars are the professional-motivated, confused, wandering-thrilled and challenging. The additional pillar is identified as trustable.This findings have been discussed in [43].

In view of the foregoing analysis as presented above, the design elements for these pillars were explored and supported by the results from PLS analysis. These results have influential relation to each other that can be used as a guideline to provide AR-based mobile learning materials for specific emotions.

Based on previous studies in KE, Partial Least Square (PLS) is an appropriate analysis to analyse the relationship of Kansei and design. Therefore, PLS was performed to identify the design elements of ARbased mobile learning materials for the Human Anatomy course relevant to the specific emotions. Conversely, it is used to determine the influential design elements in each emotion that is evoked by each specimen.

As experienced during the early phase of this research, the investigation of the design elements for 7 specimens produced 19 designs elements and 41 values. This method was use to analysed the relationship within multivariate categorical data through the item for a variable and category for the variations of a variable. With regard to PLS analysis, all these elements (item/category) were first converted into variables. Thereafter, they were combined with the average value of each representative of four main group factors that were derived from the previous analysis. This was followed by carrying out a PLS analysis to discover the relationship between emotion and design elements. The calculation of PLS Range was done to determine the influence of the elements of emotion.

Furthermore, PLS Range for every emotion representing each of the factor groups (five factors) was calculated in order to identify the influence of design including the good and bad design. The formula to calculate the PLS Range is as below:

$$
\text { PLSRange }=\text { PLSMax }- \text { PLSMin }
$$


The calculated PLS range of each design elements and value in respective emotion was presented in a form of a table. The table contains two main columns and four sub columns. The first main column is titled as Design Element, which contains Item and Category of each specimen. The second main column is Value and contains PLS value of emotion, in which PLS Range was calculated. In facilitating the calculation of the range, this PLS value was sorted in descending order for each category. The largest positive PLS value shows elements leading to a better design while the largest negative value indicates elements leading to bad design (as in Figure 2).

In this table, the arrangement of the maximum value to minimum value demonstrates the degree of adaptation of design elements that influence a certain emotion. The maximum value illustrates the suitability of the best design elements, while the minimum value illustrates the suitability of the worst design elements that influence the emotion.

Figure 2 shows the partial result of the influential design elements for the emotion of professionalmotivated. In order to visualize the sequence of dominant design elements for a specific emotion, the results are arranged in a descending order. As there is limited space in this paper, therefore only the detail data for emotion professional-motivated will be provided.

\begin{tabular}{|l|l|r|r|}
\hline \multicolumn{2}{|c|}{ Design Element } & \multicolumn{2}{c|}{ Value } \\
\hline \multicolumn{1}{|c|}{ Item } & \multicolumn{1}{|c|}{ Category } & $\begin{array}{c}\text { PLS Profesional- } \\
\text { Motivated }\end{array}$ & $\begin{array}{c}\text { PLS Range } \\
\text { (PLSMax-PLSMin) }\end{array}$ \\
\hline 1) 3D object Scale & a. 3DobjScale-Yes & 0.091278057 & 0.182556114 \\
& b. 3DobjScale-No & -0.091278057 & \\
\hline 2) Number of Colors in & a. NumCIr3DObj-More than three colors & 0.084696143 & 0.169392286 \\
3D Object & b. NumCIr3DObj-Three or less than three colors & -0.084696143 & \\
\hline 3) 3D Object Color & a. 3DobjCIrCat-Gradient & 0.084696143 & 0.169392286 \\
Category & b. 3DobjCIrCat-Solid & -0.084696143 & \\
\hline 4) Button Type & a. BtnType-Icon & 0.047554836 & 0.132699364 \\
& b. BtnType-Combination & 0.034314537 & \\
\hline c. BtnType-Label & -0.0851445528 & \\
\hline 5) Provide Information & a. PvdInfo-Simple & 0.045805772 & 0.1309503 \\
& b. PvdInfo-No Information & 0.045805772 & \\
\hline 6) Button Size & c. PvdInfo-Crowded & -0.085144528 & \\
\hline & a. BtnSize-Medium & 0.045805772 & 0.1309503 \\
& b. BtnSize-Small & 0.034966819 & \\
\hline 7) Text Font Size & c. BtnSize-Large & -0.0851445528 & \\
\hline 8) Provide Error & a. TextFntSize-Medium & 0.049145297 & 0.098290593 \\
Handling & b. TextFntSize-Small & -0.049145297 & \\
\hline 9) Provide Instruction & a. Error handlg-Yes & 0.04867579 & 0.097351579 \\
& b. Error handlg-No & -0.04867579 & \\
\hline 10) Button Grouping & a. Instruction-Yes & 0.046201536 & 0.092403073 \\
& b. Instruction-No & -0.046201536 & \\
\hline
\end{tabular}

Figure 2. A partial view of the influential design elements for emotion of Professional-Motivated

In order to design AR-based mobile learning materials, it is important that the designer sets some priorities in designing elements according to the high influence item that fits for a particular emotion. Additionally, the high influence categories for each item are equally important and should be included as a guideline. For instance, based on above result, the emotion of professional-motivated can be evoked by items such as 3D object scale, 3D object category colour, button type, and others.

Also, the Factor Analysis results show that there are four main pillars and one additional pillar of the Kansei emotions in AR-based mobile learning material for the Human Anatomy course, particularly on human heart topic. The four pillars are professionally-motivated, confused, wandering-thrilled and challenging while trustable is considered as the additional pillar as discussed in [43]. From the above presented analysis, the most suitable AR-based mobile learning material to these pillars was explored. This result can be used as a guideline to provide AR-based mobile learning materials for specific pillar, thus an enabler to discover and visualize the specimen vis a vis the emotion that have influential relation to each other.

Therefore based on the results, the design elements that have the highest influence in eliciting the first pillar (professional-motivated) has been interpreted as:

1. 3D object should be able to scale in AR.

2. 3D object should use more than three colours.

Emotional augmented reality-based mobile learning design elements: a kansei... (Fauziah Redzuan) 
3. 3D object colour should be in gradient.

4. Button type should be icon.

5. Information provided should be simple.

6. Button size should be medium.

7. Text font size should be medium.

8. Error handling should be provided.

9. Instruction should be provided.

10. The button should be grouped.

Nevertheless, the design elements in the fifth additional pillar (trustable) can also be taken into consideration as it contains some positive emotion elements that are similar to the main pillar. Among these are; text font size that should be medium and text font colour that should be one colour. Based on the listed design elements for the main pillar, the mostly type of button should be an icon and button should be grouped. However, it must be noted that the use of an icon is for easy access of information and grouping the information according to its importance [8]. As regards to the 3D object, more colours in gradient should be used. The type of 3D object to be employed should have less influence to design elements, but must be used to evoke wandering-thrilled emotion, where the type of 3D object should also be dynamic (animation). This is in line with the study of by [28].

Additionally, the Kansei concept of AR-based mobile learning materials can be generated based on the analysis of the results. This is because the primary emotions become pillars of the structure of semantic space, while secondary emotions are in with member group for each pillar. Overall, result shows that there are five pillars or factors (four main pillars and one additional pillar) that should be considered in designing AR-based mobile learning materials. In order to acquire the desired results, the designer is advisable to choose the best combination possible from a concept of emotion which may consist of one or more elements of emotion.

In conclusion, the outcome of the evaluation experiment has been described in detail in this paper. After performing statistical analysis, the final results achieved the objective for this research. As presented previously, the result of semantic space of Kansei emotion in AR-based mobile learning is obtained through PCA and FA method, whereas the design elements of AR-based mobile learning materials for specific emotions are analysed by using the PLS method.

\section{CONCLUSION}

This research has successfully achieved the objective that is to identify the specific kansei emotions associated with the AR-based mobile learning design elements. In order to achieve the objective, Kansei Engineering methodology was used because it is the most suitable method that specifically measures user's emotions evoked by products and incorporates them into a product design, in which several statistical analysis were carried out to achieve the desired result for this study.

Based on previous finding of the five factors or pillars that refer to the emotions which is the structure of kansei semantic space of AR-based mobile learning material for the Human Anatomy course. Among the five factors or pillars, four of them are main pillars while the other one is an additional pillar. The main pillars are professionally-motivated, confused, wandering-thrilled and challenging. Meanwhile, the additional pillar is trustable. Therefore, these emotions are crucial in online learning specifically in AR-based mobile learning.

The design elements of AR-based mobile learning material were derived from the result of kansei semantic space of AR-based mobile learning material that is the five factors or pillars. The findings reveal the five pillars of emotions with the corresponding five sets of design elements which have the highest influence to evoke emotions.

The findings of this research have proven that AR-based mobile learning can be designed. This indicates that educational content, including online or virtual learning material can be designed. This can be used as an emphasis on students with regard to their main focus (student centric) and align with their relevant course. In order to globalize online learning, it is important that higher educational institutions (HEIs) must stay abreast with current technology and trends with respect to the students' psychological needs. This research gives an insight to HEIs in affective designing online learning specifically AR-based mobile learning material. Thus, it is expected to form a basis for more research on online learning, particularly ARbased mobile learning materials. 


\section{ACKNOWLEDGEMENTS}

We would like to thank the Faculty of Computer and Mathematical Sciences, Universiti Teknologi MARA (UiTM), Shah Alam, Malaysia for the support to this research.

\section{REFERENCES}

[1] MOE. Executive Summary Malaysia Education Blueprint 2015-2025 .Higher Education. Volume 2025. 2015.

[2] Bawa P. Retention in Online Courses : Exploring Issues and Solutions - A Literature Review. London, England: SAGE. 2016.

[3] Hart C. Factors Associated With Student Persistence in an Online Program of Study: A Review of the Literature. Journal of Interactive Online Learning. 2012; 11(1): 19-42.

[4] Park J, Choi HJ. Factors Influencing Adult Learners' Decision to Drop Out or Persist in Online. Educational Technology \& Society. 2009;12: 207-217.

[5] EXULT. E-learning Trends. EXULT Corporation. Retrieved from www.exultcorp.com/IT/Elearning_Trends_2017_V4.pdf. 2017.

[6] Ira K, Berge Z. Online Learning's Future in the Workplace with Augmented Reality. Encyclopedia of Information Communication Technology. IGI Global. 2009.

[7] Satpute T, Pingale S, Chavan V. Augmented Reality in E-Learning Review of Prototype Designs For Usability Evaluation. International Conference on Communication, Information \& Computing Technology (ICCICT). 2015; $15-18$.

[8] Huang W, Alem L, Livingston MA. Editors. Human Factors in Augmented Reality Environment. New York: Springer. 2013.

[9] Nincarean D, Phon E, Ali MB. Collaborative Augmented Reality in Education: A Review. International Conference on Teaching and Learning in Computing and Engineering. 2014; 78-83.

[10] Solak E, Cakir R. Exploring the Effect of Materials Designed with Augmented Reality on Language Learners' Vocabulary Learning. Journal of Educators Online. 2015; 13(2): 50-72.

[11] Yen J, Tsai C, Wu, M. Augmented Reality in The Higher Education: Students Science Concept Learning and Academic Achievement in Astronomy. Procedia - Social and Behavioral Sciences. 2013; 103: 165-173.

[12] Saidin NF, Abd Halim ND, Yahaya N. A Review of Research on Augmented Reality in Education : Advantages and Applications. International Education Studies. 2015; 8(13): 1-8.

[13] Radu I, Zheng R, Golubski G, Guzdial M. Augmented Reality in the Future of Education. ACM. 2010; 1-8.

[14] Krishnan P, Vanitha V. An Integrated Framework to Enhance Performance of Online Students. Asian Journal of Research in Social Sciences and Humanities. 2016; 6: 839-855.

[15] Lim S, Lee J. An Immersive Augmented-Reality-Based e-Learning System Based on Dynamic Threshold Marker Method. ETRI. 2013; 35(6): 1048-1057.

[16] You JW. The Relationship Among College Students' Psychological Capital, Learning Empowerment, and Engagement. Learning and Individual Differences. 2016; 49: 17-24.

[17] Barker J, Gossman P. The Learning Impact of A Virtual Learning Environment: Students' Views. Teacher Education Network Journal. 2013; 5: 19-38.

[18] Krithika LB, Priya L. Student Emotion Recognition System (SERS) for E-Learning Improvement Based on Learner Concentration Metric. Procedia - Procedia Computer Science. 2016; 85(CMS): 767-776.

[19] Azuma R, Behringer R, Feiner S, Julier S, Macintyre B. Recent Advances in Augmented Reality. IEEE. 2001.

[20] Kourouthanassis PE, Boletsis C, Lekakos G. Demystifying the Design of Mobile Augmented Reality. Multimedia Tools and Applications. 2013; 1-41.

[21] Ng GW, Oon YB, Teoh EH, Yew HL. An Augmented Reality System for Biology Science Education in Malaysia. International Journal of Innovative Computing. 2016; 6(2), 8-13.

[22] Kiourexidou M, Antonopoulos N, Sgantzos M, Veglis A. Augmented Reality for the Study of Human Heart Anatomy. International Journal of Electronics Communication and Computer Engineering. 2015; 6(6): 658-663.

[23] Saidin NF, Abd Halim ND, Yahaya N. A Review of Research on Augmented Reality in Education : Advantages and Applications. International Education Studies. 2015; 8(13): 1-8.

[24] Docebo. E-Learning Market Trends and Forecast 2017-2021. Vancouver: The University of British Columbia. 2016.

[25] Satpute T, Pingale S, Chavan V. Augmented Reality in E-Learning : Review of Prototype Designs For Usability Evaluation. 2015 International Conference on Communication, Information \& Computing Technology (ICCICT). $2015 ; 15-18$.

[26] Kaufmann H. Construct3D: An Augmented Reality Application for Mathematics and Geometry Education. ACM. 2002; 656-657.

[27] Liarokapis F, Petridis P, Lister PF, White M. Multimedia Augmented Reality Interface for E-learning (MARIE). World Transactions on Engineering and Technology Education. 2002; 1(2): 173-176.

[28] Diaz C, Hincapié M, Moreno G. How the Type of Content in Educative Augmented Reality Application Affects the Learning Experience. Procedia - Procedia Computer Science. 2015; 75(Vare): 205-212.

[29] Cubillo J, Martín S, Castro M, Díaz G, Colmenar A. A Learning Environment for Augmented Reality Mobile Learning. IEEE. 2014.

[30] Jamali SS, Shiratuddin MF, Kok WW, Oskam CL. Utilising Mobile-Augmented Reality for Learning Human Anatomy. Procedia - Social and Behavioral Sciences. 2015; 197(Feb): 659-668. 
[31] Ko SM, Chang WS, Ji YG. Usability Principles for Augmented Reality Applications in a Smartphone Environment. International Journal of Human-Computer Interaction. 2013; 29(8), 501-515.

[32] Majid NA, Mohammed H, Sulaiman R. Students Perception Of Mobile Augmented Reality Applications in Learning Computer Organization. Social and Behavioral Sciences. 2015; 176: 111-116.

[33] Kourouthanassis PE, Boletsis C, Chasanidou D, Bardaki C. Tourists Responses to Mobile Augmented Reality Travel Guides: The Role of Emotions on Adoption Behavior. Amsterdam: Elsevier. 2014.

[34] Harley J, Poitras EG, Duffy M, Lajoie SP. Comparing Virtual and Location-Based Augmented Reality Mobile Learning : Emotions and Learning Outcomes. Educational Technology Research and Development. 2016; 359-388.

[35] Nagamachi M. Kansei Engineering: A New Ergonomic Consumer-Oriented Technology for Product Development. International Journal of Industrial Ergonomics. 1995; 15: 3-11.

[36] Nagamachi M. Kansei Engineering as a Powerful Consumer-Oriented Technology for Product Development. Applied Ergonomics. 2002; 33: 289-294.

[37] Lokman AM. Design \& Emotion: The Kansei Engineering Methodology. 2010; 1(1): 1-14.

[38] Schütte S. Engineering Emotional Values in Product Design - Kansei Engineering in Development. Linkoping University Institute of Technology. 2005.

[39] Lokman AM, Noor NLM, Nagamachi M. Kansei Engineering : A Study on Perception of Online Clothing Website. Proceedings of the 10th QMOD. 2000.

[40] Chuah KM, Chen C, Teh CS. Designing a Desktop Virtual Reality-Based Learning Environment with Emotional Consideration. 2011; 6 (Jan): 25-42.

[41] Redzuan F, Lokman AM, Othman ZA. Kansei Semantic Space for Emotion in Online Learning. 2014 3rd International Conference on User Science and Engineering (i-USEr 2014). IEEE. 2014; 168-173.

[42] Adnan H, Redzuan F. Evaluating Students' Emotional Response in Video-Based Learning using Kansei Engineering. 2016 4th International Conference on User Science and Engineering (i-USEr 2016). IEEE. 2016; $237-$ 242.

[43] Khairuddin ANA, Redzuan F, Daud NA. Evaluating Students' Emotional Response in Augmented Reality-Based Mobile Learning using Kansei Engineering. Communications in Computer and Information Science. 5th International Conference on User Science and Engineering (i-USEr 2018). Springer. 2018; 886: 79-89.

[44] Lokman AM, Nagamachi M. Kansei Engineering Procedures: Kansei Engineering Type II. Innovations of Kansei Engineering. New York: CRC Press. Taylor \& Francis Group. 2011.

\section{BIOGRAPHIES OF AUTHORS}

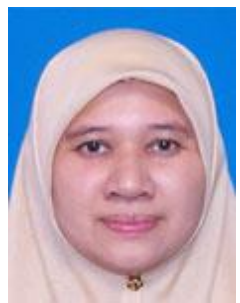

Fauziah Redzuan graduated from University of Nebraska-Lincoln, USA in 1997 and pursued her study in Master of Science in Information Technology from Universiti Sains Malaysia (USM) in 2000. She furthers her knowledge exploration and awarded Doctor of Philosophy in System Sciences and Management from Universiti Kebangsaan Malaysia (UKM) in 2017. Her current interest and research areas include Emotional/Affective E-Learning, Kansei/Affective Engineering and also Data and Database Management.

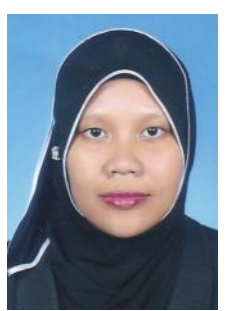

An-Nur Atiqah Khairuddin joined Sultan Idris Education University (UPSI) in 2010, as an Information Technology Officer. She received his B.S. and M.S. in Information Technology from Universiti Teknologi MARA (UiTM). Her research interests include mobile application, Kansei Engineering and Mobile Augmented Reality. She won the bronze medal of the 23th International Invention Innovation and Technology Exhibition (ITEX) in 2012. She received Best Paper Award at The 5th International Conference on User Science and Engineering (iUSEr) 2018. Currently, she is responsible for the University Integrated Management System in UPSI.

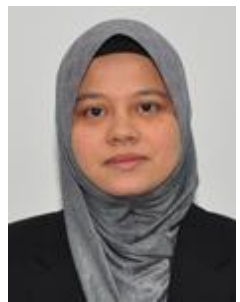

Nor Aziah Daud graduated from Universiti Utara Malaysia (UUM) in 1997 and continued her study in Master of Science in Information Technology in Universiti Teknologi MARA (UiTM) in 2003. She recently was awarded Doctor of Philosophy in Information Science from Universiti Kebangsaan Malaysia (UKM) in 2018. Her current interests are Database, Persuasive Design and E-learning. 